\title{
Posterior placoid chorioretinitis: An unusual ocular manifestation of syphilis
}

\author{
Jennifer Chen ${ }^{1,3}$ \\ Lawrence Lee ${ }^{1,2}$ \\ 'City Eye Centre, Brisbane, Australia; \\ ${ }^{2}$ Department of Ophthalmology, \\ University of Queensland, Royal \\ Brisbane Hospital, Brisbane, Australia; \\ ${ }^{3}$ Institute of Health and Biomedical \\ Innovation, Queensland University \\ of Technology, Brisbane, Australia
}

\begin{abstract}
There appears to be a re-emergence of syphilis in recent times despite a steady decline in incidence for the past decade. Diagnosis of syphilis can be clinically challenging and ocular manifestations of syphilis have a myriad of presentations and severity. Ocular syphilis can occur at any stage of the disease and may also be the only presenting sign of syphilis. We report a case of acute unilateral maculopathy, due to posterior placoid chorioretinitis associated with syphilis, in an immuno-competent patient. Ophthalmoscopy revealed a unilateral yellowish placoid lesion at the macula. Syphilis serology was positive confirming active infection. There were no other systemic signs of syphilis. The patient was treated with intravenous benzylpenicillin $1.2 \mathrm{~g}$ every four hours for two weeks. The lesion resolved with treatment and the retinal appearance returned to normal. This case highlights the importance of raising clinical suspicion of syphilis in view of unexplained decreased vision and ocular inflammation.
\end{abstract}

Keywords: syphilis, treponema, chorioretinitis, posterior uveitis, syphilitic posterior placoid chorioretinitis

\section{Introduction}

Syphilis has been described as the great 'imitator' or 'masquerade' of a myriad of ocular conditions. Diagnosis of syphilis based on ocular findings is clinically challenging, as there are no ophthalmological signs that are pathognomonic of ocular syphilis. Ocular syphilis can affect any structure of the eye and occur at any stage of the disease process, and it may also be the only presenting sign that leads to the eventual diagnosis of syphilis (Aldave et al 2001). Frequent syphilitic ocular manifestations include interstitial keratitis, chorioretinitis, retinal vasculitis, vitritis, and papillitis, among which uveitis is the most commonly reported ocular presentation of syphilis (Kiss et al 2005). Syphilitic uveitis may occur as early as six weeks after the primary inoculation and may be the only presenting systemic sign of syphilis (Gass 1997; Kiss et al 2005). Of patients with secondary syphilis, a small proportion of patients (approximately 5\%) present with syphilitic chorioretinitis and $50 \%$ of these are seen with bilateral lesions (Morgan and Laufer 1984).

\section{Case report}

A 55-year-old homosexual male presented with a 4-day history of significant visual loss in the right eye. Examination revealed visual acuity (VA) of counting fingers in the right eye and 20/15 in the left eye. His medical and family history was unremarkable and he was not taking any medication and had no known allergies. Serum glucose level was $6.9 \mathrm{mmol}$ and blood pressure was 130/70. External examination showed no signs of inflammation in the anterior segments. There was a right relative afferent pupil defect. Ophthalmoscopy revealed a large yellow-white placoid lesion at the 


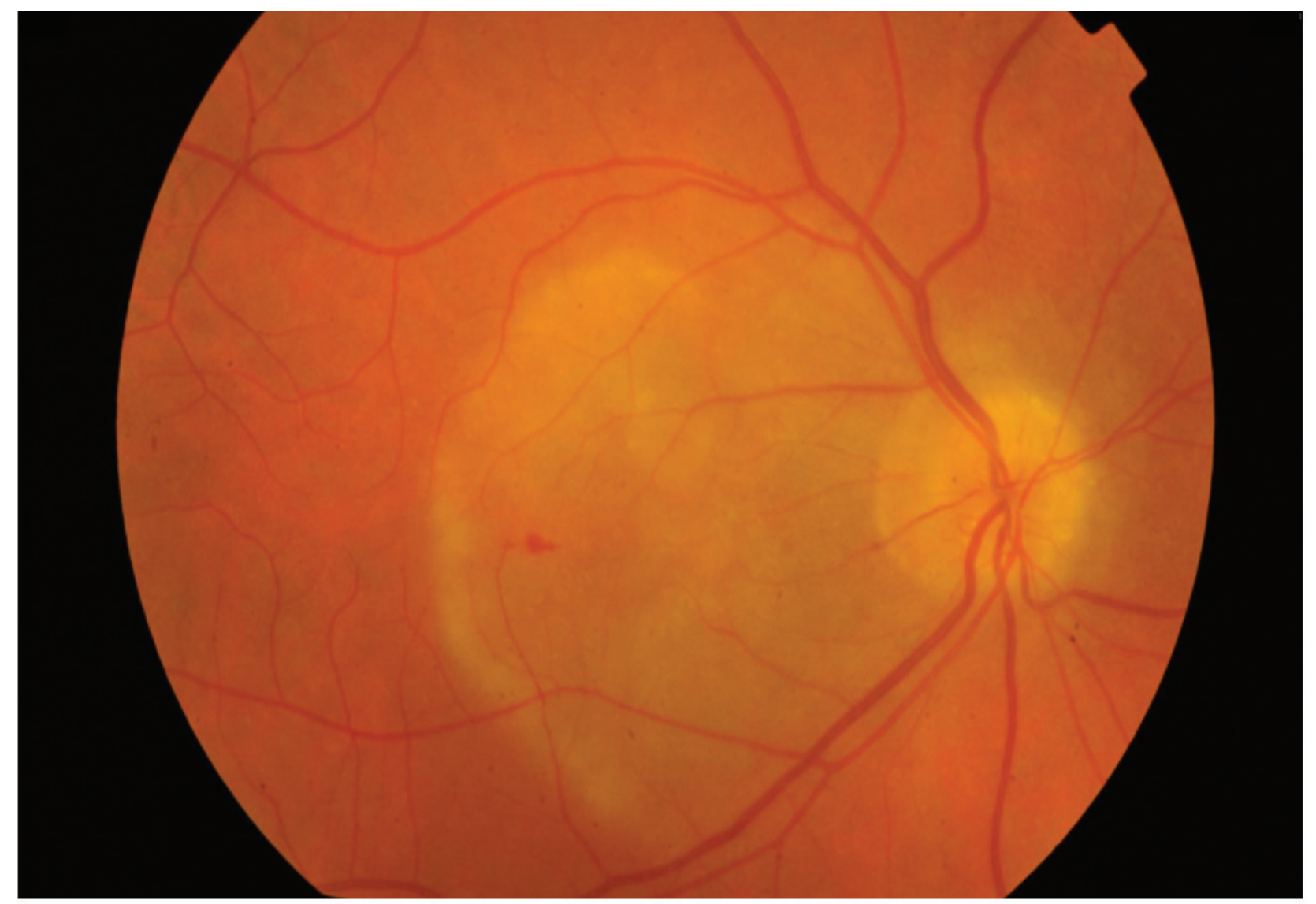

Figure I Fundus appearance of the right eye at initial presentation. There was a larger yellowish placoid lesion, with a small splinter haemorrhage in the macular region and mild vitreous inflammation.

right macula (Figure 1). There was a small splinter haemorrhage in the macular region and mild vitreous inflammation (Figure 1). Fundus fluorescein angiogram showed early hypofluoresence in the affected area, associated with late staining hyperfluorescence (Figure 2A, B). There were no signs of a choroidal neovascular membrane. Macular optical coherence tomography was also performed. There were no signs of any retinal edema or serous detachment (Figure 3). No abnormalities were detected in the left eye (Figure 4). Unilateral acute idiopathic maculopathy (UAIM) was the provisional diagnosis and the patient was asked to return for a two-week follow-up.

Two weeks following the initial presentation, VA improved to 20/30 in the right eye and there was spontaneous resolution of the vast majority of the lesion. However, at the two month follow-up, vision in the right eye had deteriorated to $20 / 40$. Screening blood tests were requested and syphilis serology revealed a rapid plasma reagin test (RPR) of 1:128 titre and positive treponemal-specific tests (Treponemal pallidum antibody, T. pallidum particle agglutination) confirming active infection. Cerebrospinal fluid (CSF) RPR was positive confirming neurosyphilis. The patient was HIV negative and his HIV status remained negative three months following initial presentation. Other screening tests including $\mathrm{C}$-reactive protein (CRP), angiotensin-converting enzyme (ACE), and full blood count (FBC) were negative, apart from a slightly elevated erythrocyte sedimentation rate (ESR) of $18 \mathrm{~mm} / \mathrm{hr}$. All other autoimmune screening tests were negative. A diagnosis of syphilitic posterior placoid chorioretinitis was made and the patient was treated with a two-week course of intravenous benzylpenicillin, $1.2 \mathrm{~g}$ every four hours. The patient did not have any systemic symptoms of syphilis such as malaise, headache, nausea or constipation. There were no other systemic manifestations such as chancres, condylomalata, macular papular rash, or lymphadenopathy.

One month following the commencement of intravenous benzylpenicillin, vision in the right eye remained at 20/25 with clearing of the vitreous cellular activity. Three months after the treatment, the RPR titre reduced from 1:128 to 1:32. The patient was prescribed with Prednefrin Forte and Acular to aid the resolution of any remaining vitreous inflammation. 

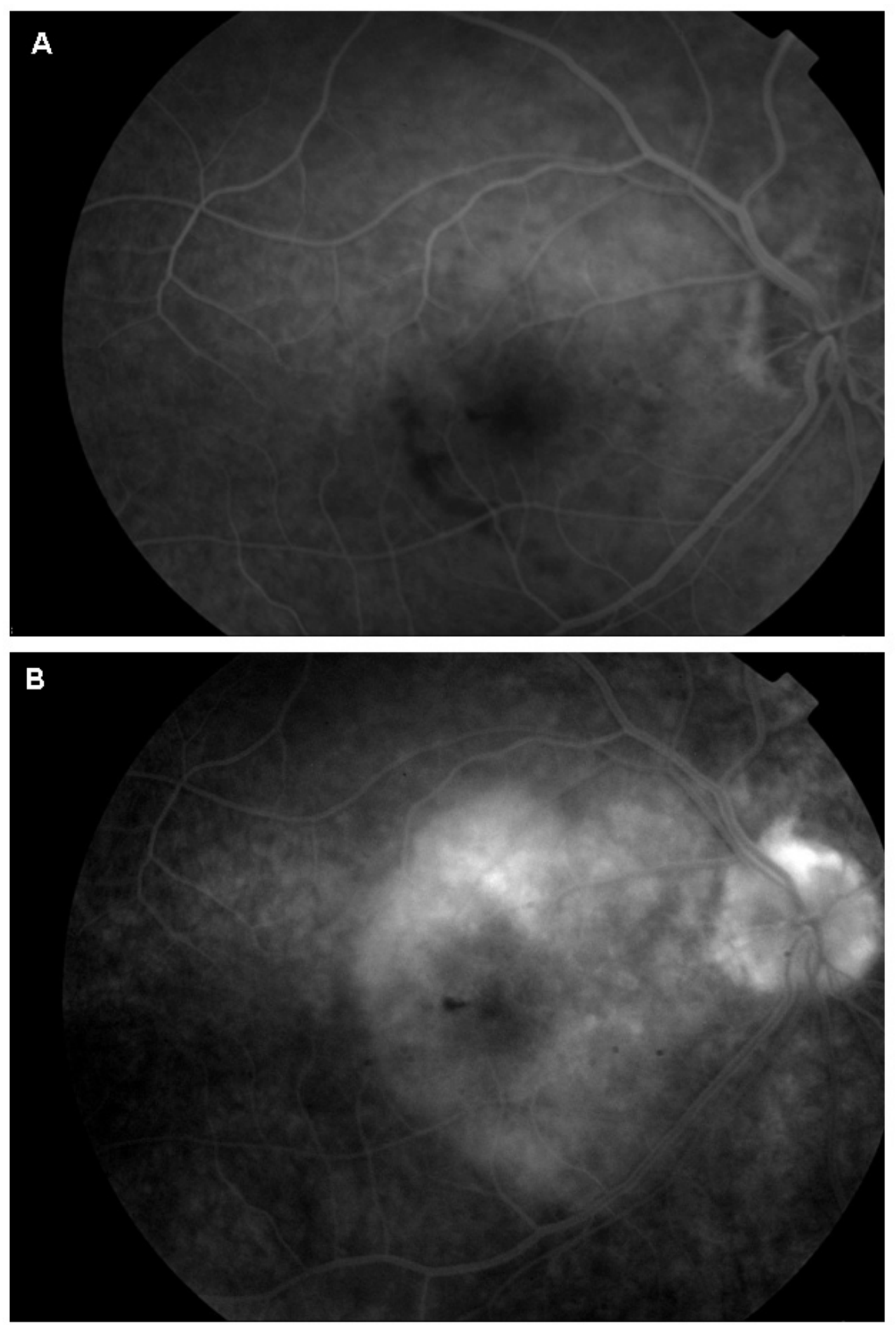

Figure 2 A: Fundus fluorescein angiogram demonstrating early hypofluorescence in the affected area. B: There was late staining with diffuse, non-progressive hyperfluorescence. There were no signs of a choroidal neovascular membrane. 


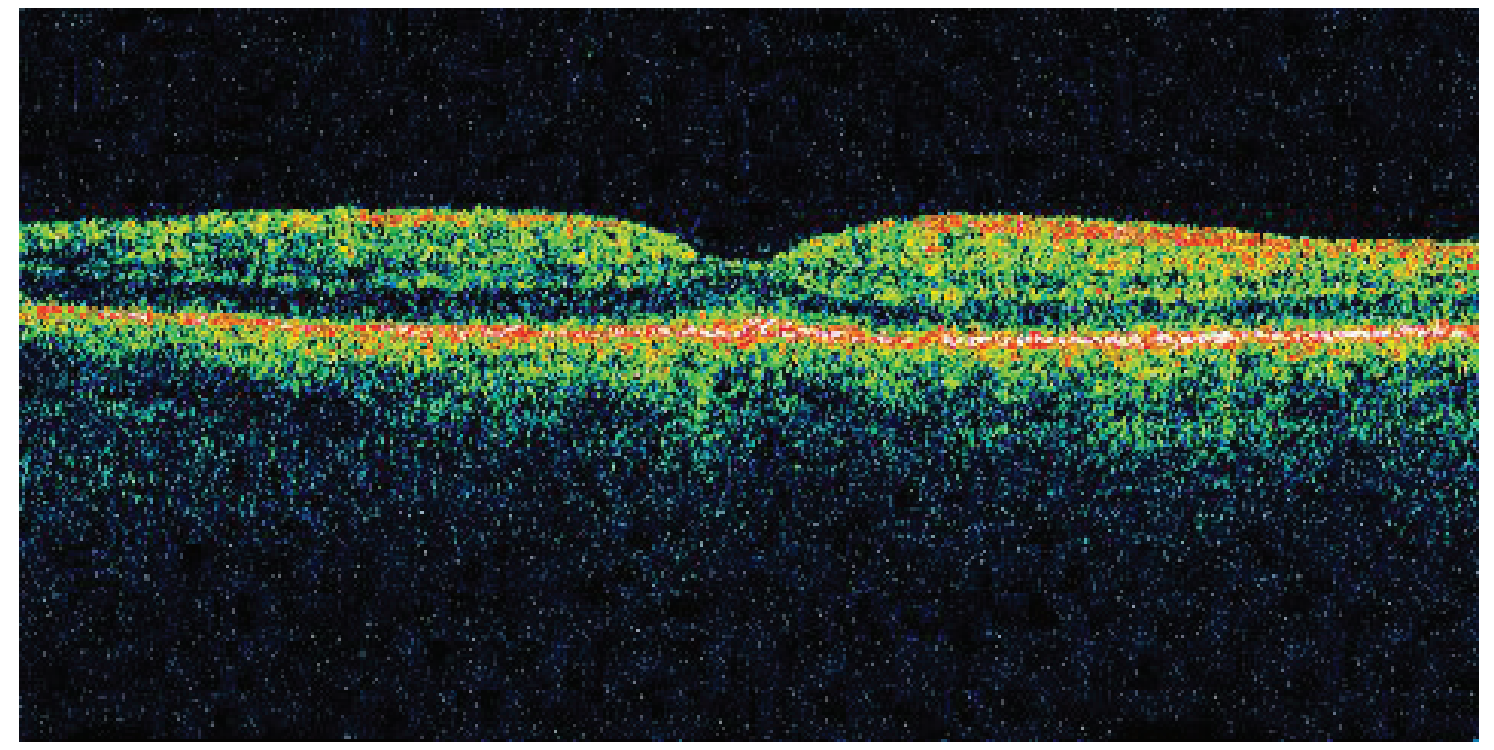

Figure $3 \mathrm{An} \mathrm{OCT} \mathrm{scan} \mathrm{of} \mathrm{the} \mathrm{right} \mathrm{eye} \mathrm{at} \mathrm{initial} \mathrm{presentation.} \mathrm{There} \mathrm{was} \mathrm{some} \mathrm{thickening} \mathrm{of} \mathrm{the} \mathrm{RPE} \mathrm{layer} \mathrm{but} \mathrm{there} \mathrm{were} \mathrm{no} \mathrm{signs} \mathrm{of} \mathrm{any} \mathrm{retinal} \mathrm{oedema} \mathrm{or} \mathrm{serous} \mathrm{detachment.}$

The left eye remained unaffected. At the 5-month follow-up, VA was $20 / 25$ in the right eye and the retinal appearance had returned to normal, apart from underlying chorioretinal atrophy in the region of the previous placoid infection (Figure 5). RPR titre was less than 1:8.

\section{Discussion}

Syphilitic posterior placoid chorioretinitis, first termed by Gass and colleagues (1990), is characterized by yellowish, ill-defined, placoid lesions that are confluent in the posterior pole or mid-periphery of the fundus. These lesions usually have a faded centre and stippled hyperpigmentation of the retinal pigment epithelium (RPE) and they can coalesce to become large confluent lesions (Gass 1997; Kiss et al 2005).

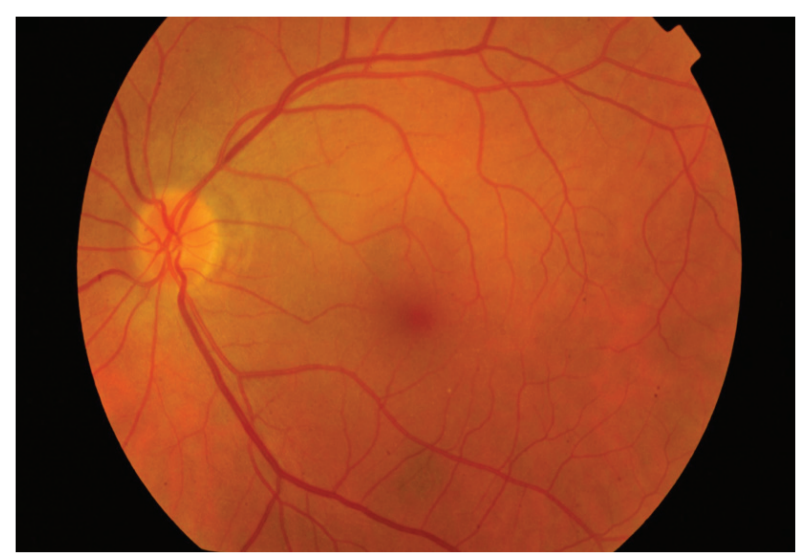

Figure 4 Fundus appearance of the unaffected left eye.
Chorioretintis is accompanied by variable amount of vitreous inflammation and may be associated with superficial haemorrhages, retinal vasculitis, disc oedema and serous detachment of the retinal pigment epithelium (Aldave et al 2001). A solitary unilateral, placoid, pale-yellow subretinal lesion is a less typical presentation of syphilitic chorioretinitis (Zamani and Garfinkel 2002).

The discrete placoid lesion consistent with syphilitic posterior placoid chorioretinitis as seen in our case is uncommon and is a manifestation typically observed among the immuno-compromised (Gass et al 1990). In our patient, there was an initial resolution of the placoid chorioretinitis

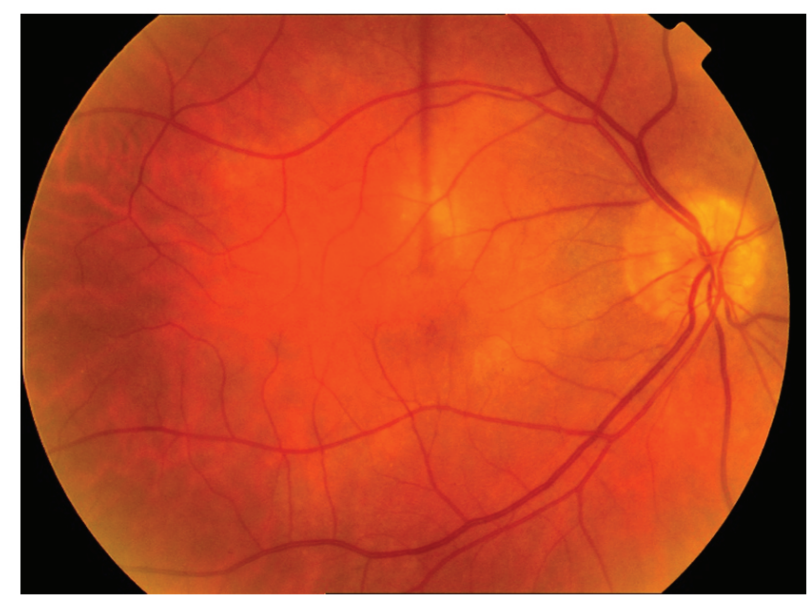

Figure 5 Fundus appearance at 5-month follow-up. The retina had returned to normal apart from underlying chorioretinal atrophy in the region of the previous placoid infection. 
and improvement of vision two weeks following the initial presentation, prior to treatment with benzylpenicillin. The initial resolution of the lesion, apparent improvement in vision and the lack of systemic signs were reasons which led to a provisional diagnosis of UAIM syndrome and resulted in a delayed diagnosis and treatment for syphilis. Other differential diagnoses included central serous retinopathy, viral retinitis, and Vogt-Koyanagi-Harada syndrome. The spontaneous resolution may represent the immunological reaction of the patient to syphilis, as there is suggestion that the clinical presentation of syphilitic posterior placoid chorioretinitis is modulated by the immune status of the patient (Zamani and Garfinkel 2002). In patients who are co-infected with HIV, syphilis may be accelerated and neurosyphilis may occur earlier due to their immune status (Aldave et al 2001). Therefore it is important that patients diagnosed with ocular syphilis should also be tested for HIV.

There appears to be a recent re-emergent epidemic of syphilis in many countries including the UK (French 2007), USA (Kerani et al 2007), Europe (French 2007), and China (Chen et al 2007), despite a steady decline in incidence for the past decade. Our case documents an unusual presentation of ocular syphilis with unilateral posterior placoid chorioretinitis in the absence of any systemic signs in an immunocompetent patient and it highlights the importance of raising clinical suspicion of syphilis in view of unexplained decreased vision and ocular inflammation. The clinical course of syphilitic eye disease is variable and in some cases the chorioretintis could resolve spontaneously while others may result in widespread atrophy and loss of retinal function, even with treatment. Given the recent increased outbreaks of syphilis, general physicians as well as eye care practitioners will have an important role to play in the diagnosis and prompt institution of appropriate treatment of this potentially fatal disease by being aware of the wide varied presentations of ocular syphilis.

\section{Disclosure}

The authors have no proprietary interest or financial support.

\section{References}

Aldave AJ, King JA, Cunningham Jnr, ET. 2001. Ocular syphilis. Curr Opin Ophthalmol, 12:433-41.

Chen ZQ, Zhang GC, Gong XD, et al. 2007. Syphilis in China: results of a national surveillance programme. Lancet, 369(9556):132-8.

French P. 2007. Syphilis. BMJ, 334(7585):143-7.

Gass JD, Braunstein RA, Chenoweth RG. 1990. Acute syphilitic posterior placoid chorioretinitis. Ophthalmology, 97:1288-97.

Gass JD. 1997. Stereoscopic atlas of macular diseases. Missouri, MosbyYear Book, Inc.

Kerani RP, Handsfield HH, Stenger MS, et al. 2007. Rising rates of syphilis in the era of syphilis elimination. Sex Transm Dis, 34:154-61.

Kiss S, Damico FM, Young LH. 2005. Ocular manifestations and treatment of syphilis. Semin Ophthalmol, 20:161-7.

Morgan S, Laufer H. 1984. Atypical syphilitic chorioretinitis and vasculitis. Retina, 4:225-31.

Zamani M, Garfinkel RA. 2002. Corticosteroid-induced modulation of acute syphilitic posterior placoid chorioretinitis. Am J Ophthalmol, 135:891-3. 
